\title{
Relação entre desempenho funcional de crianças com paralisia cerebral e qualidade de vida relacionada à saúde de seus cuidadores
}

\author{
Relationship between functional performance of children with cerebral palsy and \\ health-related quality of life of their caregivers
}

Ana Paula Rocha', Daniella Romany Viana Afonso', Rosane Luzia de Souza Morais ${ }^{2}$

Estudo desenvolvido no Depto. de Fisioterapia da Faculdade de Ciências Biológicas e da Saúde da UFVJM - Universidade Federal dos Vales do Jequitinhonha e Mucuri, campus II, Diamantina, MG Brasil

1 Graduandas em Fisioterapia na UFVJM

2 Profas. Ms. Assistentes do Depto. de Fisioterapia da UFVJM

\section{ENDEREÇO PARA}

CORRESPONDÊNCIA

Rosane L.S. Morais R.Grupiara $147 \mathrm{~A}$ bairro Purquéria 39100-000 Diamantina MG e-mail: rosanemorais@gmail.com
APRESENTAÇÃO jul. 2008

ACEITO PARA PUBLICAÇÃO set. 2008
Resumo: Cuidadores de crianças com paralisia cerebral (PC), ao lidar com as incapacidades da criança, são suscetíveis a detrimento de sua saúde física e bem-estar psicológico, o que pode ter impacto em sua qualidade de vida relacionada à saúde $(\mathrm{QV})$. O objetivo deste estudo foi verificar se há correlação entre o desempenho funcional de crianças com PC e a QV de seus cuidadores, verificando também eventual correlação entre o nível de função motora e o desempenho funcional em crianças com PC. A QV de 17 cuidadores de crianças com PC foi avaliada pelo questionário Short-Form Health Survey (SF-36); a função motora e o desempenho funcional das respectivas crianças foram avaliados pelo Gross Motor Function Classification System (GMFCS) e pelo Inventário de Avaliação Pediátrica de Disfunção (PEDI). Os dados foram tratados estatisticamente. Nenhuma correlação significativa foi encontrada entre a classificação no GMFCS ou o escore no PEDI e os escores dos cuidadores no SF-36. Foi encontrada forte correlação negativa entre os escores das crianças no PEDI e os níveis do GMFCS. A qualidade de vida de cuidadores de crianças com PC não foi pois influenciada pelo grau de limitação funcional de suas crianças. No entanto, o GMFCS mostrou-se um bom preditor de funcionalidade em crianças com paralisia cerebral.

Descritores: Criança; Cuidadores; Paralisia cerebral; Qualidade de vida

ABSTRACT: When dealing with the child's disabilities, caregivers of children with cerebral palsy (CP) are likely to experience physical or psychological suffering, which may impact their health-related quality of life $(\mathrm{QoL})$. This study searched for a correlation between CP children's functional performance and their caregivers' QoL, also looking for a correlation between gross motor functional level and functional performance in children with CP. The QoL of 17 caregivers of children with CP was assessed by the Short-Form Health Survey (SF-36); children's functional performance was evaluated by means of the Gross Motor Function Classification System (GMFCS) and the Pediatric Evaluation of Disability Inventory (PEDI). Data were statistically analysed. No significant correlation was found between children's GMFCS classification or PEDI scores and their caregivers scores in SF-36. A negative, strong correlation was found between scores at PEDI and GMFCS. The quality of life of caregivers of children with CP was hence not influenced by the children's level of functional limitation. GMFCS has shown to be a good predictor of functional performance in children with cerebral palsy.

Key words: Caregivers; Cerebral palsy; Child; Quality of life 


\section{INTRODUÇÃO}

A paralisia cerebral (PC) é uma das mais comuns desordens infantis, com uma incidência de 2 a 2,5:1000 nascidos vivos nos países desenvolvidos $^{1}$. No Brasil não existe pesquisa específica a respeito da incidência de portadores de deficiência física, sensorial ou mental ${ }^{2}$. Porém, Piovesana et al. ${ }^{3}$ relatam que, em países subdesenvolvidos, em cada 1.000 crianças, 7 são portadoras de PC.

Essa doença consiste em uma desordem do movimento, tônus e postura. Não é de caráter progressivo, mas alterações físicas e psicológicas podem ser observadas durante todo o seu curso. Prejuízos sensoriais, cognitivos e de linguagem aparecem em adição a dificuldades do aprendizado e problemas de comportamento ${ }^{4}$. Comprometimentos como espasticidade, fraqueza muscular e instabilidade postural resultam em dificuldades na marcha em 90\% das crianças com PC. Além dos comprometimentos supracitados, algumas crianças também podem ter complexas limitações de mobilidade e autocuidado, tais como as relativas a alimentação, vestuário e higiene ${ }^{1,5}$.

Segundo Raina et al. ${ }^{1}$, os cuidadores de crianças com PC geralmente têm múltiplas responsabilidades na abordagem das incapacidades da criança. Um dos principais desafios para os pais é abordar efetivamente seus problemas crônicos de saúde, enquanto mantêm as exigências da vida diária. Em alguns casos, esses cuidados podem trazer detrimento à saúde física e bem-estar psicológico dos pais, com impacto nos rendimentos e nas funções da família. Em alguns casos, o principal cuidador da criança com PC não é nenhum dos pais mas pessoas próximas, como por exemplo avó ou babá. Dessa forma, Tuna et al. ${ }^{6}$ definem cuidador principal como aquela pessoa responsável pelo cuidado direto e diário da criança, podendo ser a mãe, pai, avó, ou qualquer outra pessoa que exerça esse papel.

Qualidade de vida é definida pela Organização Mundial de Saúde como a percepção do indivíduo de sua posição na vida, contexto e valores cul- turais nos quais vive, e a relação com suas metas, objetivos e expectativas. É um amplo conceito que incorpora a saúde física da pessoa, status psicológico, nível de independência, relacionamento, inserção social e sua relação com as características do ambiente ${ }^{7}$. Qualidade de vida relacionada à saúde (QVRS) refere-se à saúde e ao bem-estar do indivíduo nos domínios físico, psicológico, social e espiritual ${ }^{8}$.

Estudos têm focalizado a relação da qualidade de vida de pais e cuidadores com as deficiências e a função motora grossa de suas respectivas crianças $^{4,6,9,10}$. Utilizam o Sistema de Classificação Funcional de Função Motora Ampla (Gross Motor Function Classification System, GMFCS) para verificação da função motora grossa, mas poucos estudos, de fato, verificam a relação entre QVRS de cuidadores e desempenho funcional em crianças com PC por meio de instrumentos padronizados. Além disso, na literatura pesquisada não existem estudos com população brasileira estabelecendo relação entre a qualidade de vida de cuidadores de PC com a limitação funcional de suas crianças.

O objetivo do estudo foi pois verificar se há relação entre desempenho funcional de crianças com PC e QVRS de seus cuidadores, residentes em uma cidade no interior do Brasil (Diamantina, MG).

\section{METODOLOGIA}

Este é um estudo de tipo transversal. Participaram do estudo todas as crianças com PC cadastradas nos sete núcleos do PSF - Programa Saúde da Família e no Núcleo de Reabilitação Nossa Senhora da Saúde de Diamantina, MG, bem como seus respectivos cuidadores. O critério de inclusão do participante adulto foi ser cuidador principal de uma criança de 6 meses a 12 anos de idade com diagnóstico médico de PC estabelecido e registrado em prontuário, residente na sede do município de Diamantina.

Para caracterização da amostra, os cuidadores forneceram dados de identificação: nome, endereço, idade, sexo e outras informações como parentesco com a criança, grau de escolaridade e ocupação. As características socioeconômicas dos cuidadores foram definidas conforme critério proposto pela Associação Brasileira de Institutos de Pesquisa de Mercado, utilizando categorias que variam de $\mathrm{A}$, indicando nível socioeconômico elevado, a $E$, indicando nível socioeconômico muito baixo, sendo as intermediárias - B, C e D - indicativas de níveis médioalto, médio e médio-baixo ${ }^{11}$.

\section{Instrumentos}

Os instrumentos usados na pesquisa foram o Inventário de Avaliação Pediátrica de Disfunção (PEDI - Pediatric Evaluation of Disability Inventory) ${ }^{12}$, para avaliar desempenho funcional; o questionário conhecido como SF-36, Medical Outcomes Study 36-item Short-Form Health Survey ${ }^{13}$, para avaliação da QVRS; e o GMFCS ${ }^{14}$, para avaliação da função motora grossa.

O PEDI é uma escala pediátrica norte-americana traduzida para o português e adaptada para contemplar as especificidades socioculturais do Brasil $^{12}$. Trata-se de um teste padronizado baseado em julgamento, realizado por meio de entrevista estruturada com os pais ou responsáveis que possam informar sobre o desempenho funcional em atividades da rotina diária da criança, e visa caracterizar aspectos funcionais na faixa entre seis meses e sete anos e meio de idade ${ }^{12}$. Estudos confirmam que o PEDI é uma avaliação válida e confiável das atividades diárias de crianças com incapacidade $^{15}$. Esse teste é dividido em três partes, que informam sobre três áreas de desempenho funcional. A primeira parte documenta as habilidades funcionais da criança nas escalas de autocuidado, mobilidade e função social; a somatória dos escores obtidos em cada escala resulta em um escore total bruto para cada uma das três áreas de habilidades funcionais. No entanto, para os objetivos do estudo, conforme instruções do manual, o escore bruto foi convertido em escore contínuo. Para este estudo foi utilizada apenas a primeira parte. 
O SF-36 é um questionário genérico de avaliação da qualidade de vida traduzido e adaptado para a população brasileira ${ }^{13}$. Nesse questionário, que pode ser respondido por pessoas a partir de 12 anos de idade, são mensurados oito domínios: função física, função social, limitações em papéis sociais devido a problemas físicos e a problemas emocionais, saúde mental, energia/ vitalidade, dor e percepção de saúde mental ${ }^{16}$. Apresenta um escore final de 0 a 100, no qual zero corresponde ao pior estado geral e 100 à percepção de melhor qualidade de vida relacionada à saúde ${ }^{16,17}$.

Também foi utilizado o GMFCS, que é um sistema de classificação da função motora ampla proposto por Palisano et al. ${ }^{14}$ e é especificamente usado para crianças com PC até 12 $a_{n o s}{ }^{4}$. A gravidade do comprometimento neuromotor é descrita, principalmente, na forma da locomoção utilizada pela criança com PC. As crianças são classificadas em cinco níveis: os níveis I e II são atribuídos a crianças que andam sem restrições; o nível III, àquelas que andam com auxílio ou suporte; no nível IV, a criança utiliza tecnologia assistiva para mover-se; e, no nível $V$, é gravemente limitada na mobilidade, mesmo com o uso de tecnologia assistiva ${ }^{18}$. Devido ao fato de as funções motoras em crianças dependerem da idade, essas foram definidas de acordo com quatro diferentes grupos: menor que 2 anos, entre 2 e 4 anos, 4 a 6 anos e 6 a 12 anos $^{4}$.

\section{Procedimentos}

Os participantes foram recrutados por meio da consulta às fichas cadastrais em todos os núcleos do PSF e no Núcleo de Reabilitação Nossa Senhora da Saúde. Os dados de todos os pacientes com necessidades especiais foram coletados e, dentre estes, foram selecionadas todas as famílias que possuíam crianças entre 6 meses e 12 anos com diagnóstico de PC estabelecido.

As famílias selecionadas (do PSF e do Núcleo de Reabilitação) foram visitadas em suas respectivas residências para esclarecimento quanto ao objetivo da pesquisa, procedimentos, bene- fícios e riscos. Todos os responsáveis pelas crianças autorizaram a participação e assinaram o Termo de Consentimento Livre e Esclarecido (TCLE). Cada cuidador também autorizou sua própria participação assinando o TCLE e preenchendo a ficha dos dados de identificação. Posteriormente, em uma nova visita, foram aplicados os instrumentos GMFCS, PEDI e o questionário SF-36. Os instrumentos foram aplicados por pesquisadoras devidamente treinadas, sendo que uma aplicava o PEDI e a outra, o SF-36 e o GMFCS. Os dois primeiros foram aplicados por entrevista com o cuidador principal; a classificação do GMFCS foi obtida pela observação do comportamento motor da criança. Este estudo foi aprovado pelo Comitê de Ética em Pesquisa da Universidade Federal dos Vales do Jequitinhonha e Mucuri.

\section{Análise de dados}

Para a análise dos dados foi utilizado o pacote estatístico SPSS (versão 15.0). Foi feita análise descritiva para caracterização da amostra. Inicialmente, as

Tabela 1 Características das crianças $(n=17)$

Tabela 2 Características das/os cuidadoras/es $(\mathrm{n}=17)$

\begin{tabular}{|c|c|c|c|c|c|c|c|c|c|c|c|}
\hline Criança & Sexo & Idade & GMFCS & Co-morbidades & Cuidador & Sexo & Idade & Parentesco & Escolaridade & Ocupação & Nível S-E \\
\hline 1 & M & 11 & I & & 1 & $\mathrm{~F}$ & 51 & Avó & PI & Diarista & D \\
\hline 2 & $\mathrm{~F}$ & 06 & I & RM/Outros & 2 & $\mathrm{~F}$ & 39 & Mãe & PC & Cozinheira & $\mathrm{D}$ \\
\hline 3 & $M$ & 11 & IV & $\mathrm{RM}$ & 3 & $\mathrm{~F}$ & 54 & Mãe & $\mathrm{PI}$ & Do lar & $\mathrm{E}$ \\
\hline 4 & $\mathrm{~F}$ & 02 & V & RM & 4 & $\mathrm{~F}$ & 28 & Mãe & EMI & Do lar & C \\
\hline 5 & $\mathrm{~F}$ & 10 & V & & 5 & $\mathrm{~F}$ & 14 & Babá & GC & Estudante/Babá & $\mathrm{C}$ \\
\hline 6 & M & 08 & V & RM /Outros & 6 & $\mathrm{~F}$ & 31 & Mãe & GI & Do lar & E \\
\hline 7 & M & 08 & II & Epilepsia/ RM & 7 & $\mathrm{~F}$ & 25 & Mãe & $\mathrm{Gl}$ & Do lar & E \\
\hline 8 & $M$ & 09 & V & & 8 & $\mathrm{~F}$ & 15 & Irmã & EMI & Estudante & D \\
\hline 9 & $\mathrm{~F}$ & 08 & V & RM /Outros & 9 & $\mathrm{~F}$ & 29 & Mãe & $\mathrm{Gl}$ & Do lar & $\mathrm{D}$ \\
\hline 10 & $\mathrm{~F}$ & 11 & V & RM/Outros & 10 & $\mathrm{~F}$ & 45 & Mãe & Gl & Diarista & $\mathrm{C}$ \\
\hline 11 & $M$ & 06 & II & & 11 & $\mathrm{~F}$ & 26 & Mãe & A & Do lar & $\mathrm{E}$ \\
\hline 12 & $\mathrm{~F}$ & 04 & V & RM/Outros & 12 & $\mathrm{~F}$ & 20 & Babá & EMI & Estudante/Babá & $\mathrm{D}$ \\
\hline 13 & $\mathrm{~F}$ & 04 & IV & RM /Outros & 13 & M & 81 & Pai & PC & Aposentado & $\mathrm{D}$ \\
\hline 14 & M & 12 & IV & RM & 14 & $\mathrm{~F}$ & 39 & $\begin{array}{c}\text { Mãe } \\
\text { adotiva }\end{array}$ & $\mathrm{Gl}$ & Do lar & E \\
\hline 15 & M & 11 & V & Epilepsia/ RM & 15 & $\mathrm{~F}$ & 29 & Mãe & EMC & Doméstica & D \\
\hline 16 & $\mathrm{~F}$ & 11 & IV & RM & 16 & $\mathrm{~F}$ & 45 & Mãe & GI & Do lar & $\mathrm{C}$ \\
\hline 17 & M & 04 & V & & 17 & $\mathrm{~F}$ & 29 & Mãe & GC & Do lar & C \\
\hline
\end{tabular}

$\mathrm{F}=$ Feminino; $\mathrm{M}=$ Masculino; GMFCS = Nível no Gross Motor Function Classification System; RM = Retardo mental; Outros = Deficit visual e/ou auditivo
$\mathrm{F}=$ Feminino; $\mathrm{M}=$ Masculino; $\mathrm{A}=$ Analfabeto; $\mathrm{PI}=$ Primário incompleto; $\mathrm{PC}=$ Primário completo; $\mathrm{GI}=$ Ginasial incompleto; $\mathrm{GC}=$ Ginasial completo; $\mathrm{EMI}=$ Ensino médio incompleto; $\mathrm{EMC}=$ Ensino médio completo; Nível S-E = Nível socioeconômico: $\mathrm{C}$ = médio; $\mathrm{D}=$ médio-baixo; $\mathrm{E}=$ pobre 
variáveis foram analisadas quanto à normalidade (pelo teste Shapiro-Wilk) e à homogeneidade de variância (Levine). Apenas três das onze variáveis analisadas apresentavam distribuição normal. Portanto, deu-se seguimento à análise inferencial com a correlação não-paramétrica de Spearman. O nível de significância adotado foi de $\alpha=0,05$.

\section{RESULTADOS}

Participaram do estudo 17 crianças e seus principais cuidadores. Nove crianças (53\%) eram do sexo masculino e oito $(47 \%)$ do sexo feminino, com idades variando de 2 a 12 $(8 \pm 3,14)$ anos. Quanto à classificação no GMFCS, duas crianças se encontravam no nível I e duas no nível II; quatro crianças foram classificadas no nível IV e nove no nível V. Essas informações, mais a indicação da presença de comorbidades associadas, podem ser visualizadas na Tabela 1 .

A maioria das cuidadoras $(64,7 \%)$ eram mães biológicas e apenas uma adotiva. A Tabela 2 mostra a idade, sexo e nível socioeconômico das cuidadoras.

A Tabela 3 apresenta os resultados da análise de correlação entre a QVRS dos cuidadores (domínios do SF-36) e o desempenho funcional das crianças (escores nas escalas do PEDI). Não houve correlação significativa.

Também foi verificado se havia correlação entre a classificação funcional das crianças (no GMFCS) e os escores dos cuidadores no SF-36 (Tabela
4). Não foi encontrada correlação significativa.

Finalmente, quanto à correlação entre função motora ampla (avaliada pelo GMFCS) e desempenho funcional da criança (nas escalas do PEDI), foi encontrada uma forte correlação negativa: com a escala de autocuidado, $r=-0,874(p=0,000)$; com a escala de mobilidade, $r=-0,911$ ( $p=0,000)$; e, com a de função social, $r=-0,797(p=0,000)$.

\section{DISCUSSÃO}

A QVRS tem sido cada vez mais utilizada para avaliação da eficácia de intervenção. Isso reflete a crescente valorização da importância de conhecer como os pacientes se sentem e o quão satisfeitos estão com o tratamento ${ }^{19}$. No caso da pediatria, é importante avaliar a QVRS dos pais ou cuidadores considerando o papel que exercem na vida das crianças. Segundo Ones et al. ${ }^{4}$, a participação e a atuação direta da família são vitais para resultados satisfatórios na reabilitação da criança com PC. No entanto, o cuidador principal, geralmente a mãe, pode sofrer estresse emocional e social.

Tuna et al. ${ }^{6}$ realizaram um estudo para investigar a QVRS de 40 cuidadores de crianças com PC em comparação com cuidadores de crianças com desenvolvimento típico, usando o SF-36. Os resultados demonstraram que os cuidadores de crianças com PC apresentaram escores do SF-36 significativamente mais baixos do que os

Tabela 3 Correlação entre os escores dos cuidadores nos domínios do SF-36 e os escores das crianças nas escalas do PEDI

\begin{tabular}{l|cc|cc|cc}
\hline Escores dos & \multicolumn{4}{c}{ Escores das crianças nas escalas do PEDI } \\
cuidadores nos & \multicolumn{2}{c}{ Autocuidado } & \multicolumn{2}{c}{ Mobilidade } & \multicolumn{1}{c}{ Função social } \\
domínios do SF-36 & $r$ & $p$ & $r$ & $p$ & $r$ & $p$ \\
\hline Capacidade funcional & 0,057 & 0,829 & $-0,226$ & 0,384 & $-0,166$ & 0,523 \\
Aspecto físico & $-0,288$ & 0,262 & $-0,323$ & 0,207 & $-0,446$ & 0,072 \\
Dor & $-0,116$ & 0,659 & $-0,324$ & 0,205 & $-0,273$ & 0,289 \\
Estado geral de saúde & 0,172 & 0,510 & 0,341 & 0,180 & 0,273 & 0,289 \\
Vitalidade & 0,346 & 0,174 & 0,212 & 0,415 & 0,124 & 0,636 \\
Aspectos sociais & $-0,174$ & 0,504 & $-0,155$ & 0,552 & $-0,196$ & 0,451 \\
Aspectos emocionais & $-0,237$ & 0,361 & $-0,269$ & 0,297 & $-0,347$ & 0,173 \\
Saúde mental & 0,002 & 0,993 & $-0,231$ & 0,372 & $-0,165$ & 0,528 \\
\hline
\end{tabular}

Tabela 4 Correlação entre os escores dos cuidadores nos domínios do SF-36 e a classificação das crianças segundo o GMFCS

\begin{tabular}{lcc}
\hline \multirow{2}{*}{ Domínio do SF-36 } & \multicolumn{2}{c}{ GMFCS } \\
& \multicolumn{1}{c}{$p$} & $p$ \\
\hline Capacidade funcional & 0,269 & 0,297 \\
Aspecto físico & 0,474 & 0,054 \\
Dor & 0,369 & 0,145 \\
Estado geral de saúde & $-0,147$ & 0,574 \\
Vitalidade & $-0,199$ & 0,443 \\
Aspectos sociais & 0,244 & 0,345 \\
Aspectos emocionais & 0,378 & 0,134 \\
Saúde mental & 0,179 & 0,492 \\
\hline
\end{tabular}

cuidadores de crianças com desenvolvimento típico.

Segundo Brehaut et al. ${ }^{20}$, mães de crianças com incapacidades físicas e psicológicas relatam mais queixa na saúde física quando comparadas às mães de crianças sem incapacidades. Além disso, os autores salientam que as mães dessas crianças estão expostas aos mesmos acontecimentos de vida e às eventuais pequenas perturbações do dia-a-dia que as outras famílias ${ }^{21}$. Por essas razões, as mães das crianças com PC poderão, comparativamente às mães de crianças sem deficiência, apresentar risco mais elevado de apresentar variações no nível da saúde mental e do bem-estar psicológico ${ }^{21}$.

No entanto, no presente estudo não foi encontrada correlação significativa entre o nível de funcionalidade de crianças com PC, avaliado pelo PEDI e pelo GMFCS, e a QVRS de seus cuidadores, a partir do SF-36. No estudo de Tuna et al. ${ }^{6}$, o nível de função motora foi investigado como um possível fator negativo de impacto na QVRS em 40 cuidadores de crianças com PC. No entanto, os autores também não encontraram correlação significativa entre desempenho motor (GMFCS) e QVRS (SF-36) dos cuidadores de crianças com PC. Ones et al. ${ }^{4}$ também não encontraram correlação entre a qualidade de vida de 46 mães de crianças com PC e os escores do GMFCS. Essa falta de correlação significante sugere que um desempenho funcional inferior não piora a qualidade de vida das mães de crianças com PC. Segundo 
os autores, é possível que a correlação tenha sido comprometida por uma distribuição heterogênea das crianças nos níveis do GMFCS, pois a maioria foi classificada nos níveis III e IV. Essa explicação poderia ser aplicada ao presente estudo, em que mais de $75 \%$ das crianças foram classificadas nos níveis IV e V.

Schneider et al. ${ }^{10}$ também investigaram a correlação entre QVRS de 30 cuidadores de crianças com PC e o desempenho funcional destas; no entanto, usaram o instrumento funcional WeeFIM, que mensura restrições ou incapacidades para realização de tarefas funcionais. Os autores encontraram uma fraca correlação positiva entre os construtos analisados $(r=0,460)$.

Qualidade de vida é um conceito multidimensional que pode ser definido como a percepção do indivíduo de sua posição na vida, no contexto da cultura e dos sistemas de valores nos quais vive e na relação com seus objetivos, expectativas, padrões e interesses ${ }^{19}$. Segundo Mugno et al. ${ }^{7}$, o nível de prejuízo na qualidade de vida das famílias de crianças com condições crônicas severas pode ser influenciado por vários fatores, tais como status socioeconômico, suporte social, características dos pais e das crianças e capacidade de enfrentamento de problemas.

King et al. ${ }^{9}$ avaliaram diversos fatores relacionados ao bem-estar de 166 pais de crianças com alterações neurológicas diversas, inclusive PC, e encontraram que o nível de escolaridade dos pais, a incapacidade e problemas de comportamento da criança, a função familiar, a satisfação com o apoio social e as estratégias de enfrentamento de problemas estão relacionados com a percepção de bem-estar dos pais.

Assim, é compreensível a falta de relação entre QVRS de cuidadores e desempenho funcional de suas crianças, encontrada no presente e em outros estudos, devido ao caráter multifatorial da qualidade de vida de um indivíduo. Além disso, Tuna et al. ${ }^{6}$ sugerem que, em vista do caráter catastrófico do nascimento de uma criança com PC, o grau de severidade da paralisia parece adquirir menor importância aos olhos de seus cuidadores.

Um outro achado do presente estudo foi a forte correlação positiva encontrada entre os escores do PEDI e os níveis do GMFCS, mostrando que a função motora grossa está relacionada com as atividades funcionais dessas crianças. Ostensjo et al. ${ }^{22}$ procuraram verificar a correlação entre os níveis do GMFCS e as três escalas do PEDI em uma amostra de 95 crianças com PC. Tal como no presente estudo, encontraram correlação positiva entre a gravidade da incapacidade motora grossa (medida pelo GMFCS) e funcionalidade nas atividades diárias das crianças (medida pelo PEDI). Os autores concluíram que o GMFCS é um ótimo preditor de funcionalidade em crianças com PC. O GMFCS e o PEDI representam aspectos diferentes, porém diretamente relacionados, da funcionalidade diária da criança com PC.

Uma das limitações deste estudo é ter sido realizado com uma apenas 17 crianças, apesar de este representar o número total de crianças com $\mathrm{PC}$, de 2 a 12 anos, registradas nas instituições pesquisadas do município de Diamantina. A literatura sugere a necessidade de se estudarem amostras populacionais em vez de centros específicos tais como clínicas e hospitais ${ }^{4,8,20}$.

\section{CONCLUSÃO}

Apesar de as crianças com paralisia cerebral demandarem mais recursos de seus cuidadores, não foi encontrada correlação entre a qualidade de vida destes e o grau de desempenho funcional das crianças, sugerindo que a qualidade de vida relacionada à saúde pode ser influenciada por diversos fatores. A forte correlação negativa entre o GMFCS e o PEDI confirma e respalda a utilização do GMFCS para avaliar funcionalidade de crianças com PC e mostra como a função motora grossa está intimamente relacionada às atividades funcionais dessas crianças.

\section{REFERÊNCIAS}

1 Raina P, O'Donnell M, Rosenbaum P, Brehaut J, Walter SD, Russell D, et al. The health and wellbeing of caregivers of children with cerebral palsy. Pediatrics. 2005;115:626-36.

2 Rotta NT. Paralisia cerebral: novas perspectivas terapêuticas. J Pediatr. 2002;78(1):48-54.

3 Piovesana AMSG, Val Filho JAC, Lima CL, Fonseca MS, Mürer AP. Encefalopatia crônica (paralisia cerebral): etiologia, classificação e tratamento clínico. In: Fonseca LF, Pianetti G, Xavier CG, editores. Compêndio de neurologia infantil. Rio de Janeiro: Medsi; 2002. p.825-38.
4 Ones K, Yilmaz E, Cetinkaya B, Caglar N. Assessment of the quality of life of mothers of children with cerebral palsy (primary caregivers). Neurorehabil Neural Repair. 2005;19(3):232-7.

5 Dodd KJ, Foley S. Partial body weight-supported treadmill training can improve walking in children with cerebral palsy: a clinical controlled trial. Dev Med Child Neurol. 2007;49:101-5.

6 Tuna H, Unalan H, Tuna F, Kokino S. Quality of life of primary caregivers of children with cerebral palsy: a controlled study with Short Form-36 questionnaire. Dev Med Child Neurol. 2004;46(9):647-8. 
7 Mugno D, Ruta L, D’Arrigo VG, Mazzone L. Impairment of quality of life in parents of children and adolescents with pervasive developmental disorder. Health Qual Life Outcomes. 2007;27:5-22.

8 Morales NMO. Avaliação transversal da qualidade de vida em crianças e adolescentes com paralisia cerebral por meio de um instrumento genérico (CHQPF50) [dissertação]. Uberlândia: Faculdade de Medicina, Universidade Federal de Uberlândia; 2005.

9 King G, King S, Rosenbaum P, Goffin R. Familycentered caregiving and well-being of parents of children with disabilities: linking process with outcome. J Pediatr Psychol. 1999;24:41-53.

10 Schneider JW, Gurucharri LM, Gutierrez AL, GaeblerSpira DJ. Health-related quality of life and functional outcome measures for children with cerebral palsy. Dev Med Child Neurol. 2001;43:601-8.

11 Abipeme - Associação Brasileira dos Institutos de Pesquisa de Mercado. Critério de classificação econômica Brasil. São Paulo; 2001. Disponível em: http://www.ufrn.br/sites/fonaprace/perfil_anexo3.doc.

12 Mancini MC. Inventário de avaliação pediátrica de disfunção: versão brasileira. Belo Horizonte: Laboratório de Atividade e Desenvolvimento Infantil; Depto. de Terapia Ocupacional, Universidade Federal de Minas Gerais; 2000.

13 Ciconelli RM, Ferraz MB, Santos W. Tradução para a língua portuguesa e validação do questionário genérico de qualidade de vida SF-36. Rev Bras Reumatol. 1999;39:143-9.

14 Palisano R, Rosenbaum P, Walter S, Russell D, Wood E, Galuppi B. Gross Motor Function Classification System for cerebral palsy. Dev Med Child Neurol. 1997;39:214-23.
15 Ostensjo S, Carlberg EB, Vollestad NK. Motor impairments in young children with cerebral palsy: relationship to gross motor function and everyday activities. Dev Med Child Neurol. 2004;46:580-9.

16 Takiuti ME, Hueb W, Hiscock SB. Quality of life after surgical myocardial revascularization, angioplasty or medical treatment. Arq Bras Cardiol. 2007;88:537-44.

17 Lemos MCD, Miyamoto ST, Valim V, Natour J. Qualidade de vida em pacientes com osteoporose: correlação entre OPAQ e SF-36. Rev Bras Reumatol. 2006;46:323-8.

18 Mancini MC, Alves ACM, Schaper C, Figueiredo EM, Sampaio RF, Coelho ZAC, et al.. Gravidade da paralisia cerebral e desempenho funcional. Rev Bras Fisioter. 2004;8:253-60.

19 Prebianchi HB. Medidas de qualidade de vida para crianças: aspectos conceituais e metodológicos. Psicol Teoria Pratica. 2003;5(1):57-69.

20 Brehaut JC, Kohen DE, Raina P, Walter SD, Russell DJ , Swinton $M$, et al. The health of primary caregivers of children with cerebral palsy: how does it compare with that of other Canadian caregivers? Pediatrics. 2004;114:182-91.

21 Monteiro M, Matos AP, Coelho R. A adaptação psicológica de mães cujos filhos apresentam paralisia cerebral: revisão da literatura. Rev Port Psicossomática. 2002;4:149-78.

22 Ostensjo S, Carlberg EB, Vollestad NK. Everyday functioning in young children with cerebral palsy: functional skills, caregiver assistance, and modifications of the environment. Dev Med Child Neurol. 2003;45:603-12. 